\title{
The Effect of Synchronized Inputs at the Single Neuron Level
}

\author{
Öjvind Bernander, Christof Koch \\ Computation and Neural Systems Program, California Institute of Technology, \\ Pasadena, CA 91125 USA
}

\author{
Marius Usher \\ Department of Psychology, Carnegie Mellon University, \\ Pittsburgh, PA 15213 USA
}

It is commonly assumed that temporal synchronization of excitatory synaptic inputs onto a single neuron increases its firing rate. We investigate here the role of synaptic synchronization for the leaky integrateand-fire neuron as well as for a biophysically and anatomically detailed compartmental model of a cortical pyramidal cell. We find that if the number of excitatory inputs, $N$, is on the same order as the number of fully synchronized inputs necessary to trigger a single action potential, $N_{\mathrm{t}}$, synchronization always increases the firing rate (for both constant and Poisson-distributed input). However, for large values of $N$ compared to $N_{\mathrm{t}}$, "overcrowding" occurs and temporal synchronization is detrimental to firing frequency. This behavior is caused by the conflicting influence of the low-pass nature of the passive dendritic membrane on the one hand and the refractory period on the other. If both temporal synchronization as well as the fraction of synchronized inputs (Murthy and Fetz 1993) is varied, synchronization is only advantageous if either $N$ or the average input frequency, $f_{\text {in, }}$ are small enough.

\section{Introduction}

It has long been postulated that the synchronous firing activity of cortical neurons is a crucial stage underlying perception. The psychologist Milner (1974) first proposed that neurons responding to a "figure" fire synchronously in time, while neurons responding to another figure or to the "ground" fire randomly: the "primitive unity of a figure" would be defined at the neuronal level by synchronized firing activity.

Several years later, von der Malsburg (1981) formulated his influential correlation theory of brain function on the basis of the importance of synchronized activity. How this theory could be used to temporally segregate patterns was demonstrated by von der Malsburg and Schneider 
(1986). Using computer simulations, they showed that from an initially totally interconnected set of tonotopic neurons two distinct groups of neurons-corresponding to two distinct voices-arise. The mechanism of this segmentation is the temporal synchronization of simultaneously active cells using a fast Hebbian synaptic modulation mechanism. This idea has been extended by Crick and Koch $(1990,1992)$, who postulated that synchronized and oscillatory firing activity in a subset of cortical neurons constitutes the neuronal correlate of visual attention and awareness.

Over the last 10 years, a small but growing community of electrophysiologists has focused on the synchronized electrical activity among two or more simultaneously recorded neurons in the cortex of cats and monkeys (Toyama et al. 1981a,b; Abeles 1982, 1990; Ts'o et al. 1986; Aertsen et al. 1989; Nelson et al. 1992; Kreiter and Singer 1992; Murthy and Fetz 1993). Remarkably, in some of these studies, cross-correlation among two cortical cells reveals a central peak with a width of less than 1 msec. These studies were brought to the forefront with the discovery (based on Freeman's earlier work; Freeman 1975) of $40 \mathrm{~Hz}$ oscillations in the visual cortex of cats (Eckhorn et al. 1988; Gray and Singer 1989) and the crucial demonstration that these oscillatory responses can become temporally synchronized in a stimulus-dependent manner (Gray et al. 1989). In the cat, the oscillations can be phase-locked with a phase-shift of $\pm 3 \mathrm{msec}$ around the origin at distances up to $7 \mathrm{~mm}$ (Engel et al. 1992). The existence and strength of the $40 \mathrm{~Hz}$ oscillations in the awake monkey appear to be highly variable (some laboratories routinely observe them while others do not). In consequence, recent theoretical studies focus on synchronized neuronal activity-rather than oscillations-as the basis for figure-ground segregation (Bush and Douglas 1991; Koch and Schuster 1992; Tononi et al. 1992).

The principal idea underlying these studies is the belief that synchronized neuronal firing in large populations of pyramidal cells causes a higher firing rate in postsynaptic target cells (after suitably accounting for axonal and synaptic delays; Manor et al. 1992; Abeles 1982). This, in fact, is already inherent in the McCullough and Pitts (1943) neuron: if one such binary "unit" has a threshold of two, the simultaneous activity of two presynaptic neurons is required to bring the unit above threshold. However, it has rarely been asked to what extent more realistic and biophysically plausible models of neurons prefer synchronized to desynchronized, excitatory synaptic input. Are there physiologically meaningful conditions under which temporally synchronized input leads to less effective postsynaptic firing than less synchronized activity? This is the question we address here and we find that under many conditions synchronized firing is not optimal for the cell in terms of generating the largest number of spikes. We shall leave aside the interesting question of how synchronized activity arises in neural populations (see Abbott 1990, 
1993; Mirrollo and Strogatz 1990; Sompolinsky et al. 1990; Hansel and Sompolinsky 1992; Usher et al. 1993).

To our knowledge, only a single paper has investigated the possible "negative" effect of synaptic synchrony on postsynaptic firing frequency (Murthy and Fetz 1993). Their numerical study varies the fraction of cells that is perfectly synchronized among each other, concluding that synchronization increases the postsynaptic firing frequency only under certain conditions. In our more general investigation, we use both an analytically treatable neuron model (from the family of integrate-and-fire models) as well as computer simulations of a biophysically very realistic cortical pyramidal cell to investigate the effect of single-shot and repetitive synaptic input at various synchronization levels. The principal-and most limiting - assumption we make here is that the dendritic tree of cells is passive and does not contain special, fast voltage-dependent nonlinearities, which are limited to the cell body.

The degree of synaptic correlation in the input varies in accordance with two independent factors: the temporal spread of synchronization, $T$, referred to as the desynchronization interval and the fraction of neurons that is synchronized, $r$. As we shall see, these two factors affect the postsynaptic firing frequency in different ways. In the first two sections of the paper, we will investigate the effect of the desynchronization interval on the firing rate, assuming that $N$ synapses are each activated only once, under two extreme assumptions: (1) the input is Poissondistributed throughout $T$ with, on average, $N$ synaptic inputs and (2) the input is constant, approximating the situation of regular input activity every $T / N$ msec. In Section 4 , we will deal with the added complication arising from repetitive synaptic input.

\section{Synchronicity in Integrate-and-Fire Models}

We will first consider different variants of the integrate-and-fire (I\&F) model neuron (Knight 1972; Fig. 1a), under the assumption that $N$ synapses are activated only once (single-shot case).

In its simplest version, discrete synaptic inputs arriving at times $t_{i}$ place an identical charge $Q_{0}$ onto a capacitance $C$, charging up the membrane potential across the capacitance by $\Delta V=Q_{0} / C$. When the voltage reaches a fixed threshold value, $V_{t}$, a point-like pulse is generated and the potential $V(t)$ is reset to 0 . Two important modifications to this model include a membrane leak conductance $G$ and an absolute refractory pe$\operatorname{riod} T_{\mathrm{rp}}$. The leaky or forgetful integrate-and-fire model has finite memory: since the membrane potential decays exponentially between synaptic inputs (with time-constant $\tau_{\mathrm{m}}=\mathrm{C} / \mathrm{G}$ ), events that occurred in the past are less effective than more recent ones. The effect of $T_{\text {rp }}$ is to hold the potential $V(t)$ to 0 for the duration $T_{\text {rp }}$ after the model has generated a spike, rendering all synaptic inputs ineffective during this time. The 

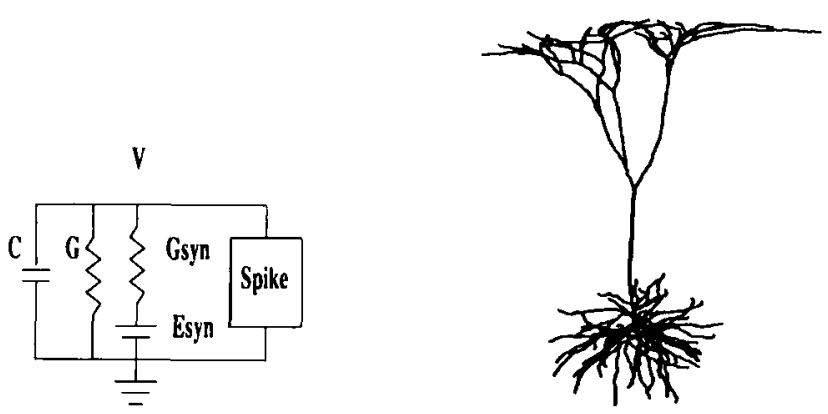

Figure 1: The two neuronal models used in our study. (a) The leaky integrateand-fire (I\&F) model with optional membrane leak $G$ and refractory period $T_{\mathrm{rp}}$. Synaptic input can be modeled either as a conductance change $G_{\text {syn }}$ (shown) or as a current source $I_{\text {syn }}$ (not shown). (b) Compartmental model of a morphologically reconstructed layer $\mathrm{V}$ pyramidal cell from cat cortex (Bernander et al. 1991), using 400 passive dendritic compartments and a soma containing eight voltage- and time-dependent Hodgkin-Huxley-like currents.

main virtue of this family of models is their simplicity, allowing us to study some of their properties analytically.

2.1 Regular Synaptic Input. We here assume that the synaptic input arrives at a constant rate $\lambda=N / T$; in other words during the interval $T$, $N$ synaptic inputs arrive in a regular manner, spaced $T / N$ msec apart. Let $T_{\text {spike }}$ be the time required to charge up the membrane from rest $(V=0)$ to $V_{\mathrm{t}}$. The total number of output spikes, $N_{\mathrm{sp}}$, generated during this interval $T$ will be the largest integer $n$ for which

$$
n T_{\text {spike }}+(n-1) T_{\text {rp }}<T,
$$

that is

$$
N_{\mathrm{sp}}=\text { Floor }\left[\frac{T+T_{\mathrm{rp}}}{T_{\text {spike }}+T_{\mathrm{rp}}}\right] \text {, }
$$

where Floor $[x]$ is the largest integer smaller than or equal to $x$. For an analytical treatment it is more convenient to use a continuous approximation:

$$
N_{\mathrm{sp}}=\frac{T+T_{\mathrm{rp}}}{T_{\text {spike }}+T_{\mathrm{rp}}} .
$$


Table 1: Analytical Expressions for the Time $T_{\text {spike }}$ Required for Constant Synaptic Input Arriving at Rate $\lambda=N / T$ to Reach Threshold, Assuming a Continuous Approximation of the Discrete Input."

\begin{tabular}{|c|c|c|c|}
\hline Case & Model & Synaptic input & $T_{\text {spike }}$ \\
\hline 1 & $I \& F$ & Current $I_{0}=C \lambda \Delta V$ & $\frac{N_{1}}{\lambda}$ \\
\hline 2 & I\&F & Conductance & $-\frac{C}{\lambda G_{x y 11}} \ln \left(1-\frac{V_{t}}{E_{\mathrm{y} n}}\right)$ \\
\hline 3 & Leaky I\&F & Current $I_{0}=C \lambda \Delta V$ & $-\tau_{m} \ln \left(1-\frac{N_{1}}{\tau_{m} \lambda}\right)$ \\
\hline 4 & Leaky I\&F & Conductance & $-\frac{C}{G+\frac{C}{\lambda G_{\mathrm{syyn}}}} \ln \left(1-\frac{V_{1} C}{\lambda E_{\mathrm{syn}} G_{\mathrm{syn}}}-\frac{V_{1}}{E_{\mathrm{syn} n}}\right)$ \\
\hline
\end{tabular}

"See Appendix A for derivation.

We also assume that $N_{t}=V_{t} / \Delta V$, that is, the number of simultaneous current inputs required to reach threshold is an integer.

The only quantity that needs to be evaluated in equation 2.3 is $T_{\text {spike. }}$. Case 1 is an integrate-and-fire model with a constant rate $\lambda=N / T$ of identical synaptic input pulses, each one dumping the charge $Q_{0}=C \Delta V$ onto the capacitance. This is equivalent to injecting the constant current $I_{0}=C \lambda \Delta V$ onto the capacitance. In the third case, the same current $I_{0}$ is injected into the leaky integrate-and-fire model. In the other two cases, the input is treated as a conductance input $G_{\text {syn }}>0$ in series with a synaptic battery $E_{\mathrm{syn}}=70 \mathrm{mV}$, for the standard (case 2) or for the leaky I\&F model (case 4). This is equivalent to a single effective conductance of value $\lambda G_{\text {syn }}$ that is activated during the interval $T$.

We derive in Appendix $\mathrm{A}$ an analytical expression for $T_{\text {spike }}$ in the fourth and most general case (see Table 1). If the synaptic conductance is small compared to the membrane leak conductance $G$, the input can be treated as fixed synaptic input current $I_{\text {syn }}=\lambda C \Delta V=\lambda E_{\text {syn }} G_{\text {syn }}$ and we arrive at $T_{\text {spike }}$ for current input (case 3). The time to spike for the standard I\&F model can be obtained by setting the leak term $G=0$. While conductance inputs are more relevant to the physiological situation where massive synaptic input fires the pyramidal cell at high rates (Bernander et al. 1991), further analysis is simplified if current inputs are used.

We evaluated equation (2.3) for the case of conductance inputs to the I\&F model with $N=1000$ inputs and either no or a fixed refractory period ( $T_{\mathrm{rp}}=2 \mathrm{msec}$; see Fig. 2 ) and used values for $G, C, T_{\mathrm{rp}}$, and $N_{\mathrm{t}}$ that mimic the values observed in cortical pyramidal cells (see below).

For the standard integrator (with $T_{\mathrm{rp}}=0$ and $G=0$; top curve), the number of output spikes, $N_{\mathrm{sp}}$, is independent of $T$. In fact, $N_{\mathrm{sp}}$ is always independent of the arrival times of the input but only depends on the total number of inputs: $N_{\mathrm{sp}}=N / N_{\mathrm{t}}$ for the I\&F model with an infinite memory. When a membrane leak $G$ is introduced, the number of output spikes decreases with $T$ because earlier inputs leak away with a time 

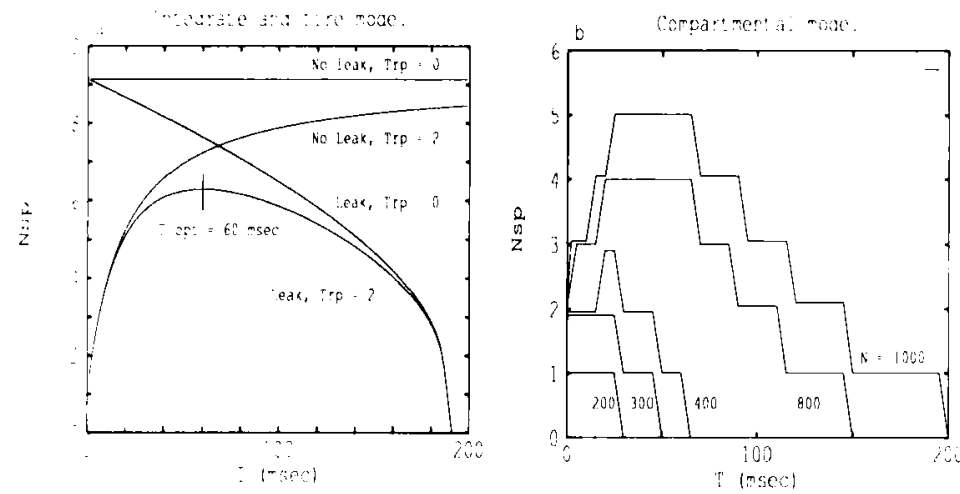

Figure 2: Temporal dispersion of synaptic input and its effect on firing rate for different single cell models. A fixed number of identical excitatory inputs $N$ is evenly distributed along the interval $T$ and the number of output spikes $N_{\text {sp }}$ is computed as a function of $T$. (a) Leaky integrate-and-fire model with $N=1000$ conductance inputs. The leak conductance $G$ is either 0 or $58.8 \mathrm{nS}$, and the refractory period $T_{\mathrm{rp}}$ is either 0 or $2 \mathrm{msec}$. The membrane leakage "pulls down" the right end of the curve, while the refractory period pulls down the left end of the curve. $\Delta V=0.25, V_{\mathrm{t}}=15 \mathrm{mV}, \tau_{\mathrm{m}}=17 \mathrm{msec}$, and $N_{\mathrm{t}}=$ 60. These parameters are similar to those of the detailed model. The optimal value of $T, T_{\text {opt }}$, is marked on the bottom curve. (b) Compartmental model of layer V pyramidal cell. $N=200-1000$ excitatory, fast AMPA synapses were distributed throughout the cell. $N_{\mathrm{t}}=66$ synchronized somatic synaptic input are required to trigger one action potential. For $N=1000$ the I\&F model with refractory period and conductance inputs is in good qualitative agreement with the compartmental model. The principal result of our study is that for $N>N_{\mathrm{t}}$ synchronization of synaptic input causes the cell to fire fewer spikes than if the synaptic input is temporally dispersed (i.e., the optimal $T>0$ ).

constant $\tau_{\mathrm{m}} \approx C / G$. This is at the heart of the traditional argument for the advantage of synchronizing synaptic input in terms of eliciting the maximum number of postsynaptic spikes: temporal dispersion of synaptic input reduce their effectiveness (e.g., Abeles 1982). However, when a refractory period, $T_{\mathrm{rp}}$ is introduced into the leaky I\&F neuron (lower curve in Fig. 2a), the initial part of the curve is "pulled down," so that for small desynchronization intervals $T, N_{\mathrm{sp}}$ will increase with $T$. The reason for this "overcrowding" effect is that synaptic input in excess of $N_{t}$ will be "wasted." Synaptic inputs arriving during the refractory period do not contribute to the excitability of the cell. Thus, for $N>N_{t}$, desynchronized synaptic input increases the spiking rate, or high synchronicity of massive synaptic input reduces firing rate. The optimal $T$ is a compromise 
between the effects of $G$ and $T_{\text {rp }}$ and is about $60 \mathrm{msec}$ for $N=1000$ as shown in Figure 2.

Substituting current inputs for conductance inputs had only a minor effect on the I\&F models. If the parameters were adjusted to give similar values of $N_{\mathrm{sp}}$ for small $T$, then current inputs give slightly larger values for large $T$ (graphs not shown).

It is conceivable that the peaked form of $N_{\mathrm{sp}}$ could be due to synaptic saturation. Rather than inputs being "wasted" during the refractory period, an increased resting potential would reduce the driving force $E_{\text {rev }}-V(t)$ for the excitatory synapses. Such saturation effects exist only if the synaptic input is treated as a conductance change and could, in principle, reduce $N_{\mathrm{sp}}$ for high input rates $\lambda=N / T$ and small values of $T$. However, $d N_{\mathrm{sp}} / d T<0$ for case 4 with $T_{\mathrm{rp}}=0 \mathrm{msec}$, independent of $E_{\mathrm{syn}}$, and therefore no peak can occur (see monotonically decreasing curve in Figure 2a).

2.2 Optimal Desynchronization Interval. How does $T_{\text {opt }}$, the optimal value of $T$, that is the desynchronization interval at which $N_{\mathrm{sp}}$ is maximized, depend on the various parameters? To find $T_{\text {opt }}$, we compute $d N_{\mathrm{sp}} / d T$ for the leaky I\&F model with current inputs and set this derivative to zero (see Appendix B). This expression cannot be solved in closed form since it is of the form $f(y)=\log (1-y)$. Instead we show the numerically obtained value of $T_{\text {opt }}$ in Figure 3 . Note that all three axes are in dimensionless variables: $T_{\mathrm{opt}} / T_{\mathrm{rp}}, T_{\mathrm{m}} / T_{\mathrm{rp}}$, and $N / N_{\mathrm{t}}$.

As can be seen, $T_{\text {opt }}$ is almost exactly linear in $N / N_{t}$, except for values of $N / N_{1}$ in the neighborhood of 1 . This is not surprising, since at high input rates $T_{\text {opt }}$ becomes much larger than $\tau_{m}$ and a dynamic steady state condition prevails during most of the single-shot. In other words, for $N \gg N_{\mathrm{t}}$ there exists an optimal input rate $f_{\text {in }}=N / T_{\text {opt }}$ (see Appendix B). For our parameter values in the leaky $I \& \mathrm{~F}$ model, this rate is approximately 10 inputs/msec. If $f_{\text {in }}$ is increased above this $10 \mathrm{kHz}$ rate, the refractory period will reduce $N_{\mathrm{sp}}$, while if $f_{\text {in }}$ is decreased below $10 \mathrm{kHz}$, the membrane leak causes a reduction in $N_{\text {sp. }}$.

$T_{\text {opt }}$ increases with $\tau_{\mathrm{m}}=C / G$ but not in a linear fashion due to the opposing effects of $T_{\mathrm{rp}}$ (favoring larger values of $T_{\mathrm{opt}}$ ) and $G$ (favoring smaller values).

2.3 Synchronicity for Poisson-Distributed Input. Up to now we considered synaptic input that arrives at a constant rate. Let us now analyze the more realistic situation of Poisson-distributed current inputs with a mean rate $\lambda=N / T$ to the leaky integrate-and-fire model over a fixed temporal interval of duration $T$.

Due to the stochastic nature of the input, the membrane potential $V$ executes a random walk-like trajectory. When the potential reaches $V_{\mathrm{t}}$ a pulse is generated and $V(t)$ is reset to zero. While the probability 


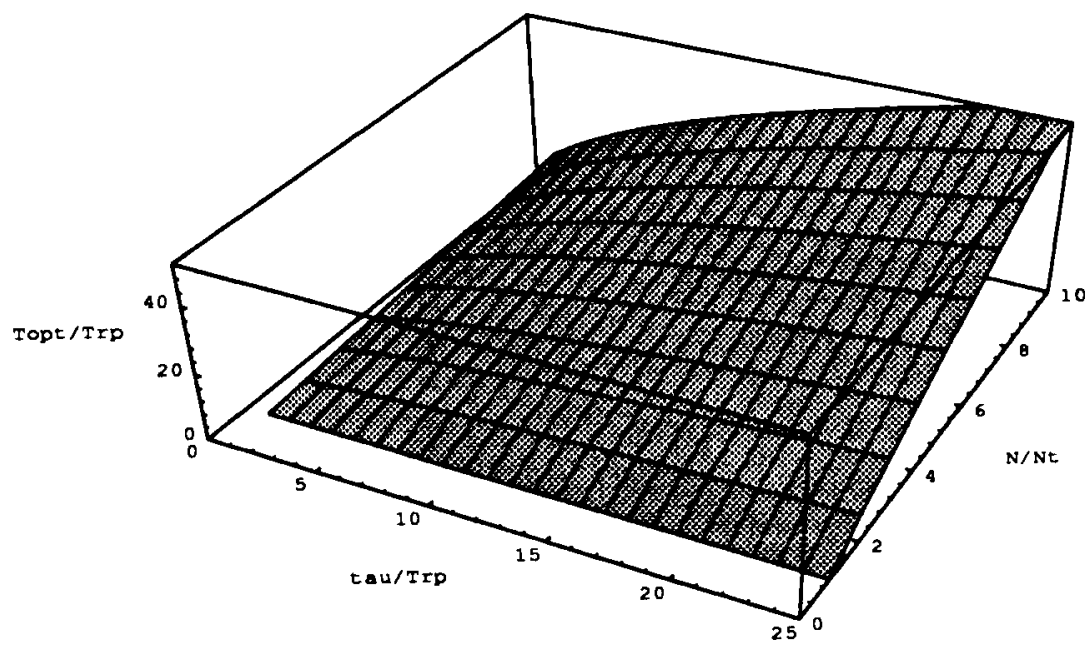

Figure 3: The optimal value of $T, T_{\text {opt }}$, in units of $T_{\text {opt }} / T_{\mathrm{rp}}$, in the leaky integrateand-fire model for current input as a function of the normalized number of inputs $N / N_{\mathrm{t}}$ and normalized time-constant $\tau_{\mathrm{m}} / T_{\mathrm{rp}} . T_{\text {opt }}$ is almost linear in $N$ for $N>>N_{\mathrm{t}}=60$; for example, if the number of inputs $N$ doubles, they should be spread out over twice as long an interval $T$ as before in order to maximize firing frequency.

distribution function for the entire interspike interval distribution is not known for the leaky I\&F model, it is possible to compute the mean time to threshold, $T_{\text {spike }}$ (known as the first time to passage problem). Following Ricciardi (1977; see Appendix C for details), we compute the mean value of $T_{\text {spike }}$ from the equilibrium probability distribution of the membrane potential by using Siegert's recursion formula:

$$
T_{\text {spike }}(\lambda)=\tau_{m} \sqrt{\pi} \int_{x_{1}(\lambda)}^{x_{2}(\lambda)} e^{z^{2}}[1+\operatorname{erf}(z)] d z
$$

where the limits of integration are

$$
\begin{aligned}
& x_{1}(\lambda)=-\sqrt{\lambda \tau_{\mathrm{m}}} \\
& x_{2}(\lambda)=\frac{N_{\mathrm{t}}-\lambda \tau_{\mathrm{m}}}{\sqrt{\lambda \tau_{\mathrm{m}}}}
\end{aligned}
$$

The average number of output spikes $N_{\mathrm{sp}}$ can now be calculated from equation 2.3 and is plotted in Figure 4 as a function of the desynchronization interval $T$. Comparing $N_{\mathrm{sp}}$ for this stochastic input with the 


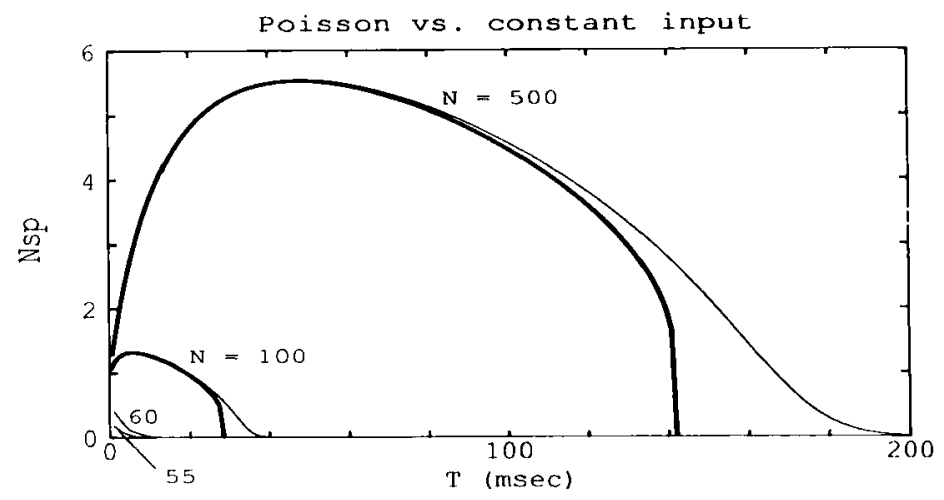

Figure 4: Number of output spikes (from equation 2.4) for Poisson-distributed (thin lines) and for constant synaptic input (thick lines) for the leaky integrateand-fire model with current input (see case 3 in Table 1) as a function of the desynchronization interval $T$. The different curves are for different values of $N$, that is, the average number of synaptic inputs arriving during the interval $T$. For $N<N_{\mathrm{t}}=60$ or for $T>T_{\text {cutoff, fluctuations in the random input can }}$ always push the potential above threshold, while the model ceases to respond to constant input. For $T<T_{\text {cutoff }}$ and $N>N_{t}$, that is, for large input rates $\lambda=N / T$, the two models agree closely. For $N \leq N_{\mathrm{t}}$, the analytical approximation deviates significantly from the numerical one due to truncation error and only the latter is shown for $N=\mathbf{5 5}$ and 60 .

superimposed functions for the constant input we can see two important differences at the two extremes of the axis. One major difference is when on average less than $N_{\mathrm{t}}$ inputs are present. For constant synaptic input, no spikes are generated for $N<N_{\mathrm{t}}=60$ and $N_{\mathrm{sp}} \neq 0$ only for $T=0$ at $N=60$. However, for Poisson-distributed input, there always exists a nonzero probability that stochastic fluctuations in the input will carry the potential above threshold. Likewise, Poisson input can in principle, for large values of $T$, always exceed the threshold $N_{\mathrm{t}}$, while this is not possible for regularly timed input (where the cutoff value of $T$ is given by $T_{\text {cutoff }}=\tau_{\mathrm{m}} N / N_{\mathrm{t}}$ ), resulting in a "tail" for large values of $T$. For all other values of $T<T_{\text {cutoff }}$ and $N>N_{t}$, the constant synaptic input closely approximates Poisson-distributed input. In other words, for large enough values of the synaptic input rate $\lambda=N / T$, Poisson input can be approximated by constant input (case 3 in Table 1). 


\section{Synchronicity in a Detailed Model of a Pyramidal Cell}

To what extent are our results due to the very simple neuronal model we have been using? After all, I\&F neurons have a fixed threshold, no dendritic tree, no voltage- and time-dependent conductances, and no synaptic dynamics. In order to address this issue, we simulated a detailed compartmental model of a morphologically reconstructed layer 5 pyramidal cell from visual cortex in the anesthetized adult cat (Douglas et al. 1991; see Fig. 1b; for details of the model see Bernander et al. 1991, 1992).

Briefly, the dendritic tree consists of about 400 passive compartments (with $C_{\mathrm{m}}=1 \mu \mathrm{F} / \mathrm{cm}^{2}, R_{\mathrm{m}}=100 \mathrm{k} \Omega-\mathrm{cm}^{2}, R_{\mathrm{i}}=200 \Omega-\mathrm{cm}$, and $E_{\text {rest }}=$ $-66 \mathrm{mV}$ ). The membrane resistance and reversal potential for each compartment is adjusted to mimic a $0.5 \mathrm{~Hz}$ "background" activation of 4000 excitatory, fast AMPA (or non-NMDA) synapses and 1000 GABAergic inhibitory synapses. Since each synapse corresponds to a small conductance change, the effective membrane resistance is reduced to $10-50 \mathrm{k} \Omega$ $\mathrm{cm}^{2}$. The soma contains 8 active currents, including two sodium currents $\left(I_{\mathrm{Na}}\right.$ and $\left.I_{\mathrm{Na}, \mathrm{p}}\right)$, four potassium currents, one of which is calciumdependent $\left(I_{\mathrm{DR}}, I_{\mathrm{A}}, I_{\mathrm{M}}\right.$, and $\left.I_{\mathrm{AH}}\right)$, one calcium current $\left(I_{\mathrm{Ca}}\right)$, and an anomalous rectifier $\left(I_{\mathrm{AR}}\right)$. The $A$ current allows the cell to spike at low frequencies over an extended range and the combination of $I_{\mathrm{Ca}}$ and $I_{\mathrm{AHP}}$ causes the firing rate to adapt by a factor 2-3 (for medium and high firing rates). The input resistance in the presence of synaptic background activity is $15 \mathrm{M} \Omega$ and the time constant $17 \mathrm{msec}$. The peak voltage of the spikes drops below $0 \mathrm{mV}$ at approximately $500 \mathrm{~Hz}$, suggesting an absolute refractory period of about $2 \mathrm{msec}$.

A simulation was run on the full model for $N=200$ to 1000 fast, excitatory, voltage-independent AMPA synapses distributed throughout the dendritic arbor in accordance with the known anatomical distribution (Fig. 2b). For $N=1000$ the same basic effect is observed as in the leaky I\&F model with refractory period. In the case of total synchronization, $T=0$, only two spikes are produced due to overcrowding. A maximum of 5 spikes is obtained for $T=25-65 \mathrm{msec}$ and the response decreases to 0 spikes for $T=200 \mathrm{msec}$. When $N$ is reduced to 300 synapses or less, the peak in $N_{\mathrm{sp}}$ disappears. Note, however, that now $N_{\mathrm{sp}}$ is essentially flat around the origin, implying that the cell is not highly tuned to small $T$ (for the $\tau_{m}=17 \mathrm{msec}$ used here).

To assess to what extent this behavior is due to the fact that synaptic input increases the membrane conductance-rather than injecting a current into the cell-we approximated this condition by reducing the synaptic conductance change $G_{\max }$ for each synapse by a factor 10 , while increasing the driving force $E_{\text {rev }}-V$ by a factor 10 . This removed any saturation in the dendritic tree, and thus more current was injected during stimulation (curves not shown). The main difference was that a few 
more spikes were obtained at every $T$, while the $N_{\mathrm{sp}}$ still peaked for approximately the same values of $T$.

Substituting NMDA synapses for half of the AMPA synapses had the effect of broadening the peak, as well as making it less pronounced. This can be explained by the much slower time course of the NMDA synapses $\left(\tau_{\mathrm{m}_{\text {divay }}}=40 \mathrm{msec}\right.$ ), which is conceptually similar to desynchronizing the much faster AMPA synapses. No obvious cooperative effects were seen due to the negative input conductance of the NMDA synapses.

\section{Correlated Synaptic Input}

In the previous sections, we assumed that $N$ independent synapses were each activated only once (single-shot case). However, synaptic input is repetitive (cells fire more than once) and can be correlated. How are our previous results affected by such correlated activity? The degree of correlation in the input may vary in accordance with two factors: the temporal spread of synchronization ( $T$ as expressed by the width of the cross-covariance function between input neurons), and the fraction of neurons, $r$, that is synchronized. By varying each of the factors independently, one can interpolate between a fully synchronized and a fully desynchronized input.

If each of the $N$ input neurons is firing with a Poisson probability distribution with mean rate $f_{\text {in, }}$, two extreme situations can be considered:

- If none of the neurons is correlated $(r=0)$, the input consists of a single Poisson process of events of height $\Delta V$ and rate $\lambda=N f_{\text {in }}$. The mean interspike interval and output firing rate can then be estimated from equation 2.4 and will be denoted by $f_{\text {out.0 }}$.

- When all $(r=1)$ neurons are perfectly correlated $(T=0)$, the input is equivalent to a single Poisson stream of events of height $N \Delta V$ and rate $\lambda=f_{\text {in }}$. Assuming $N>N_{t}$ (otherwise the neuron will rarely fire), each synchronized event triggers one spike (the refractory period prevents multiple spikes for such synchronized input currents) and the output frequency is equal to the input frequency $f_{\text {in }}$.

The intermediate situation ( $r N$ neurons perfectly correlated with $T=0$, and the rest independent) interpolates between these two limiting cases as shown in Figure 5 . Thus increasing the number of perfectly synchronized and correlated neurons is advantageous only if the response in the uncorrelated case is lower than $f_{\text {in, }}$ that is if $f_{\text {out }, 0}\left(N, f_{\text {in }}\right)<f_{\text {in }}$. We derive the border between these two domains by finding those values of $N$ and $f_{\text {in }}$ where this inequality turns into an equality. As we saw in Section 2.3, if $\lambda$ is large enough, the value of $T_{\text {spike }}$ for Poisson input is well approximated by that of constant input given by case 3 in Table 1 . Using this 

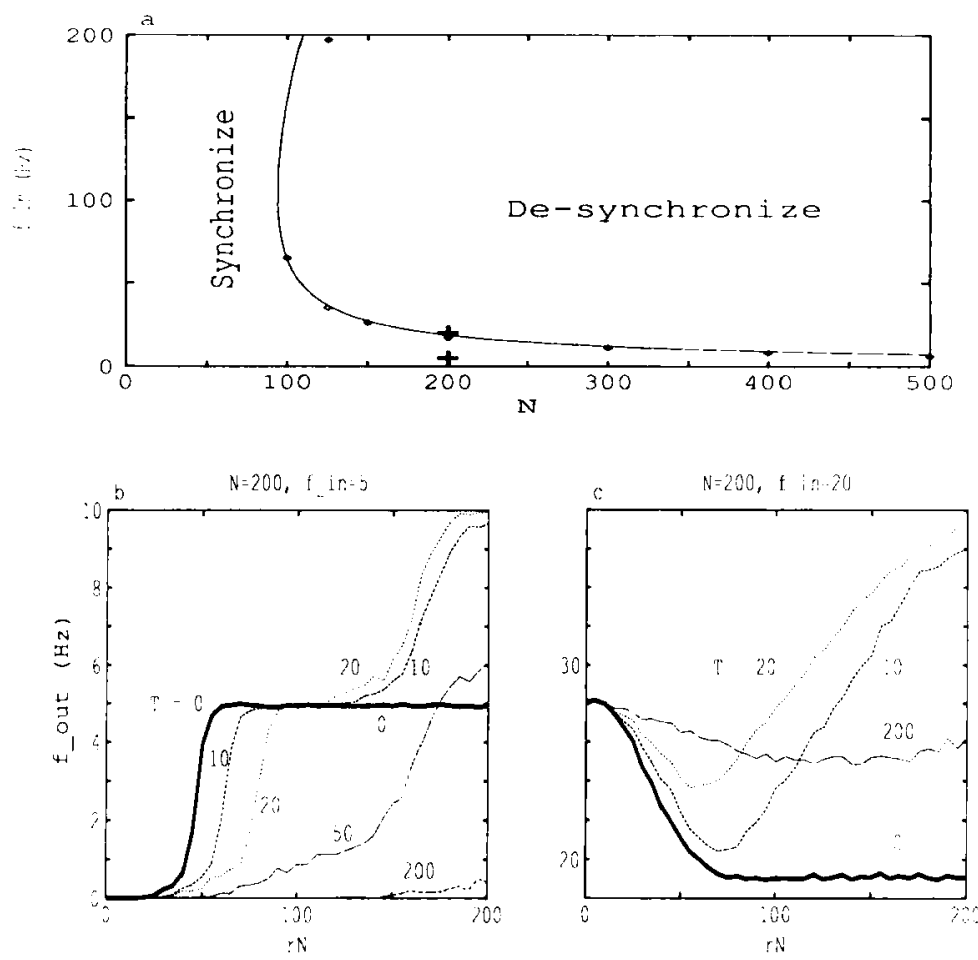

Figure 5: Simulation results for the response rate $f_{\text {out }}$ of a leaky I\&F neuron with current input ( $\tau_{\mathrm{m}}=17 \mathrm{msec}, T_{\mathrm{rp}}=2 \mathrm{msec}$, and $\left.N_{\mathrm{t}}=60\right)$ to a varying fraction $r N$ of correlated inputs out of a total population of $N=200$ input neurons. The bold curves in (b) and (c) highlight the response when the correlated neurons are perfectly synchronized $(T=0)$, while the thin lines correspond to nonzero values of the desynchronization interval $T$ as indicated. (a) Border in the $f_{\text {in }}-N$ plane delimiting the parameter range for which perfect synchronization at $T=0$ reduces the firing rate from the opposing situation (see equation 4.1, diamonds represent simulation results). For small values of either $N$ (as long as $N>N_{\mathrm{t}}$ ) or $f_{\text {in }}$ synchronization always increases $f_{\text {out }}$. (b) For $f_{\text {in }}=5 \mathrm{~Hz}$, synchronization always increases the firing frequency. (c) For larger values of the input frequency, here $f_{\text {in }}=20 \mathrm{~Hz}$, too much or too little temporal synchronization decreases $f_{\text {sut }}$.

latter result as well as $f_{\text {out } .0}=1 /\left(T_{\text {spike }}+T_{\mathrm{rp}}\right)$ and $\lambda=N f_{\text {in }}$ we arrive at

$$
N=\frac{N_{\mathrm{t}}}{\tau_{\mathrm{m}} f_{\mathrm{in}}} \times \frac{1}{1-e^{-\left(1 / f_{\mathrm{in}}-T_{\mathrm{rp}}\right) / \tau_{\mathrm{m}}}}
$$


This expression, then, demarcates the two domains. For values of $N$ and $f_{\text {in }}$ below and to the left of this border (Fig. 5a), increasing the number $r N$ of correlated neurons enhances the output rate while above and to the right of this curve increased synchronization reduces the output rate. The latter effect is due to the fact that at higher $N$ and $f_{\text {in }}$, input spikes are rendered ineffective due to the refractory period.

For low values of the input frequency relative to the leak term, that is, when $f_{\text {in }} \ll 1 / \tau_{m}$, the $f_{\text {in }}$ term in the exponent in the right hand of equation 4.1 side can be neglected, leading to an inverse relationship between $N$ and $f_{\text {in }}$ and to a hyperbolic curve for small values of $f_{\text {in }}$ in Figure 5a.

What effect does the temporal width of the cross-covariance function $T$ have on postsynaptic firing frequency? We approached this question by numerically evaluating $f_{\text {out }}$ for a variety of different settings in the relevant four-dimensional space spanned by $r, T, N$, and $f_{\text {in. }}$. In the simulations shown in Figure $5 b$,c, we compute $f_{\text {out }}$ as a function of the number of synchronized inputs using the leaky I\&F model with current inputs.

Figure $5 \mathrm{~b}$ shows the response rate for a population of $N=200$ input neurons, all firing with a mean input rate $f_{\text {in }}=5 \mathrm{~Hz}$. We are here well in the domain (see the lower cross in Fig. $5 a$ ) where perfect $(T=0)$ synchronization will lead to an increase in the firing rate. If none of the inputs is correlated, we are in the first of the limiting cases discussed at the beginning of the section with $f_{\text {tut }, 0}=0$. As $r N$ increases, the unit starts to fire. At perfect levels of temporal synchronization, $f_{\text {wut }}$ steeply rises in the neighborhood of $r N \approx N_{\mathrm{t}}$ and saturates for large values of $r N$ at the firing rate $f_{\text {in }}$ (second limiting case discussed above), since all 200 inputs fire at once, causing only a single postsynaptic spike per input volley. For a finite desynchronization interval, here $T=10$ and $20 \mathrm{msec}$, the steep rise in $f_{\text {out }}$ occurs at somewhat larger values of $r \mathrm{~N}$ than for perfect synchronization. However, as $r$ continues to increase, the firing rate increases to almost twice the frequency compared to perfectly synchronized input $(T=0)$, expressing the fact that two spikes are fired on average per input volley (due to the temporal spread of all 200 inputs over 10 or $20 \mathrm{msec}$ ). For larger desynchronization intervals (here 50 and $200 \mathrm{msec}$ ), the leaky membrane limits the response of the I\&F unit and the postsynaptic response remains small.

Figure $5 \mathrm{c}$ illustrates the reverse case when the input rate is so high (here $f_{\text {in }}=20 \mathrm{~Hz}$ ), that temporal synchronization leads to a reduction in postsynaptic response (see the upper cross in panel a). Here, increasing the fraction of perfectly synchronized neurons $r N$ causes a drop in $f_{\text {out }}$, except when a $T=10 \mathrm{msec}$ desynchronization interval is being used for large values of $r N$. The fact that when all neurons are correlated at the endpoints $r N=200$ of Figure $5 b, c$, the optimal desynchronization interval is between 10 and $20 \mathrm{msec}$ and can be understood from our analysis of the single-shot case in Section 2. 


\section{Discussion}

A number of proposals for linking neuronal firing with higher-level "emergent" properties explicitly or implicitly assume that synaptic input synchronization always leads to an increase in postsynaptic firing frequency compared to the desynchronized case (see the Introduction). We here investigate this hypothesis in detail. Before we summarize and interpret our results, let us state the principal limitations of our study. We investigated the firing properties of two distinct neuronal models: the analytically treatable integrate-and-fire (I\&F) family of integrator models (Knight 1972) as well as a biophysically detailed compartmental model of an anatomically reconstructed cortical pyramidal cell (Bernander et al. 1991, 1992). This model assumes that no voltage-dependent membrane currents are present in the dendrites (with the exception of the voltage-dependent NMDA synaptic input) and that the normal complement of ionic currents (eight in our model) generates the responses seen in a typical, regular-firing pyramidal cell (McCormick et al. 1985; Connors and Gutnick 1990). We did not consider bursting cells that can generate two or more fast spikes in response to an appropriate synaptic input nor voltage-dependent sodium or calcium currents in the dendritic tree (Llinás 1988). Both situations render any analysis such as the one carried out here considerably more complex. Sufficiently fast and strong dendritic nonlinearities, such as postulated by Softky (1994), can in principle render the cell susceptible to specific temporal arrangements of synaptic input (i.e., specific values of $T$ ) and would invalidate our analysis. Given that Softky and Koch (1993) postulate such dendritic nonlinearities to explain the high variability of the firing rate of cortical pyramidal cells, we plan to investigate the dependency of such a cell to synchronized synaptic input in a later study. We here provide the baseline against which the performance of more complex neuronal models need to be evaluated.

We assume that synaptic input is either constant or distributed according to a Poisson process. Our detailed analysis of the power spectrum, interspike interval distribution and firing variability of nonbursting cortical cells in the awake and behaving monkey firing at high rates supports the Poisson hypothesis (Softky and Koch 1993; Bair et al. 1993). Finally, we here consider only the effect of excitatory synaptic input, neglecting the effect of synchronization of inhibitory synaptic input. However, in as far as steady-state conditions are met (i.e., for large enough values of $\lambda$ ), the current due to the inhibitory synaptic input can be subtracted from the excitatory current, yielding a net effective input current (or input rate) and all of our arguments apply.

Both the I\&F models as well as the detailed biophysical model display the same behavior: if the entire excitatory synaptic input is correlated ( $r=1$; single-shot case), temporal synchronization (small values of $T$ ) increases the output firing rate only if the average number of spikes (as characterized by a Poisson process with rate $\lambda=N / T$ ) is lower than 
the number of input $N_{\mathrm{t}}$ needed to reach threshold (Fig. 2). ${ }^{1}$ For rates significantly larger than $N_{\mathrm{t}} / T$, there will be a nonzero, optimal desynchronization interval. As witnessed in Figure 3, this optimum interval increases linearly with $N / N_{\mathrm{t}}$ and sublinearly with $\tau_{\mathrm{m}} / T_{\mathrm{rp}}$. For desynchronization intervals larger than $T_{\text {opt }}$, the response is reduced due to temporal dispersion induced by the membrane leak, while the refractory period limits the usefulness of high synchronization. Any neuronal model with a refractory period will display such a tendency against overcrowding of synaptic inputs. It should be noted that such overcrowding can occur at what is believed to be physiological levels of synaptic input; 1000 synaptic inputs (constituting about $10 \%$ of all excitatory synaptic inputs; Larkman 1991) impinging onto our pyramidal cell within $50 \mathrm{msec}$ give rise to twice the number of spikes than the same number of inputs applied instantaneously $(T=0)$.

Because the similarity between the I\&F and the detailed models, we only use the former when we investigate the more complex situation arising during repetitive input when only a fraction $r$ of the $N$ input synapses is correlated (with the width of the central peak in the cross-covariance function characterized by $T$ ). This is the situation Murthy and Fetz (1993) studied, assuming always $T=0$. They conclude that synchronization is useful only when $N, \Delta V$, or $f_{\text {in }}$ are not too large. We explicitly found (Fig. 5a) the domain in $N . f_{\text {in }}$ space for which correlated inputs enhance the response. For values of $f_{\text {in }}$ and $N$ below the border displayed in Figure $5 \mathrm{a}$, and if the number of correlated inputs $r N<N_{\mathrm{t}}\left(N_{\mathrm{t}}\right.$ is about 60 as estimated by biophysical parameters from our reconstructed neuron) perfect temporal synchronization (with a zero-width peak) is advantageous (Fig. 5a). In this regime, the assumption that high levels of firing synchronization-as expressed by sharp peaks in the cross-covariance function-play a significant role in various perceptual processes is valid (Milner 1974; von der Malsburg 1981; Abeles 1982, 1990; Gray et al. 1989; Crick and Koch 1990; Kreiter and Singer 1992). If $r N>N_{t}$, perfect synchronization ceases to be optimal due to overcrowding. In this regime, small enough values of the average input frequency $f_{\text {in }}$ in combination with small desynchronization intervals $(T \approx 10-20 \mathrm{msec}$; Fig. $5 \mathrm{~b}, \mathrm{c}$ ) enhances the response rate compared to perfect or no temporal synchronization. Thus, cross-covariance functions with pronounced but wide peaks can indeed be more advantageous than extremely narrow central peaks in the cross-covariance (e.g., cell pairs of the $T$ type in Nelson et al. 1992). We conclude that (in the absence of fast and powerful active dendritic conductances) if the synchronization of the firing of cortical cells is indeed a crucial signal underlying higher-level perceptual processes,

\footnotetext{
'We would like to note in passing that both in this study and in our analysis of the firing variability, characterized in terms of both the variability of the number of spikes and the variability of the interspike intervals (Softky and Koch, 1993), the leaky integrate-and-fire model (Knight 1972) is in good qualitative agreement with the biophysically detailed pyramidal cell model with passive dendrites.
} 
the brain must take care to ensure that only some minimal number of neurons are simultaneously active.

Appendix A: $T_{\text {spike }}$ for Constant Inputs in the Modified I\&F Model

We derive here the time $T_{\text {spike }}$ it takes for a constant conductance input, $\lambda G_{\text {syn }}$ to charge the membrane from 0 to $V_{\mathrm{t}}$ in the leaky integrate-and-fire model (case 4 in Table 1).

The input is approximated by a constant synaptic conductance increase in series with a synaptic battery $E_{\text {syn }}$ (here, $E_{\text {syn }} G_{\text {syn }}=C \Delta V$ ). Replacing the two parallel conductances $G$ and $\lambda G_{\text {syn }}$ with a single equivalent conductance $G^{\prime}=G+\lambda G_{\text {syn }}$ and replacing the battery $E_{\text {syn }}$ with $E^{\prime}=E_{\text {syn }} \lambda G_{\text {syn }} / G^{\prime}$ we arrive at a first-order, ordinary differential equation:

$$
C \frac{d V}{d t}+\left(V-E^{\prime}\right) G^{\prime}=0
$$

Solving this and setting $V$ to $V_{\mathrm{t}}$ and $t=T_{\text {spike }}$ leads to

$$
T_{\text {spike }}=\frac{C}{G^{\prime}} \ln \left(1-\frac{V_{\mathrm{t}}}{E^{\prime}}\right)
$$

which can be rewritten as

$$
T_{\text {spike }}=-\frac{C}{G+\lambda G_{\text {syn }}} \ln \left(1-\frac{V_{\mathrm{t}} G}{\lambda E_{\mathrm{syn}} G_{\mathrm{syn}}}-\frac{V_{\mathrm{t}}}{E_{\mathrm{syn}}}\right)
$$

The expression for current inputs (case 3 ) can be obtained as a limiting case by setting $I_{0}=\lambda C \Delta V=\lambda E_{\text {syn }} G_{\text {syn }}$ and letting $G_{\text {syn }} \rightarrow 0$ and $E_{\text {syn }} \rightarrow$ $\infty$, keeping $G_{\text {syn }} E_{\text {syn }}$ constant. $T_{\text {spike }}$ for the nonleaky $I \& F$ model (cases 1 and 2) can be simply obtained by setting the membrane leak $G$ to 0 [and exploiting $\ln (1+d x)=d x$ for small values of $d x]$.

\section{Appendix B: Optimal Desynchronization Interval}

For current inputs, the number of output spikes is

$$
N_{\mathrm{sp}}(T)=\frac{T+T_{\mathrm{rp}}}{T_{\mathrm{rp}}-\tau_{\mathrm{m}} \log \left[1-\left(N_{\mathrm{t}} T / \tau_{\mathrm{m}} N\right)\right]}
$$

where $N_{\mathrm{t}}=V_{\mathrm{t}} / \Delta V$ is the number of simultaneous EPSPs necessary to reach threshold. If we define the dimensionless variables $a_{1}=N / N_{1} \cdot a_{2}=$ $\tau_{\mathrm{m}} / T_{\mathrm{rp}}$, and $y=N_{\mathrm{t}} T /\left(N \tau_{\mathrm{m}}\right)$, take the derivative of equation B.1 and set the resulting expression to zero, we obtain

$$
\frac{y+\left(1 / a_{1} a_{2}\right)}{1-y}=\frac{1}{a_{2}}-\log (1-y)
$$


Note that $y$ depends only on the two dimensionless quantities $a_{1}$ and $a_{2}$. No closed-form expression exists for the solution of $y\left(a_{1}, a_{2}\right)$, which was therefore solved numerically. When $y\left(a_{1}, a_{2}\right)$ is graphed (not shown), it is almost independent of $a_{1}$ except for small values of $a_{1}$. Because of the linear relationship between $T_{\text {opt }}$ and $y$, the optimal desynchronization interval $T_{\text {opt }}$ is linear in $N$ for most parameter values, given constant $\tau_{m}$ and $T_{\mathrm{rp}}$.

\section{Appendix C: Poisson Inputs}

We here present a brief derivation of the mean first-passage time in equation 2.4 (for details see Ricciardi 1977). If a leaky integrator with time constant $\tau_{\mathrm{m}}$ receives a stream of Poisson-distributed EPSPs of rate $\lambda$ and depolarization $\Delta V$, the transition probability density function satisfies the following equation:

$$
f(V . t+\Delta t \mid U, t)=[1-\lambda \Delta t] \delta\left(U_{0}-V\right)+\lambda \Delta t \delta\left(U_{1}-V\right)
$$

with

$$
U_{0}=U-U \frac{\Delta t}{\tau_{\mathrm{m}}}
$$

and

$$
U_{1}=U-U \frac{\Delta t_{1}}{\tau_{\mathrm{m}}}+\Delta V-\left(U-U \frac{\Delta t_{1}}{\tau_{\mathrm{m}}}+\Delta V\right) \frac{\Delta t_{2}}{\tau_{\mathrm{m}}}
$$

The first term is associated with relaxation of the membrane potential if no input reaches the cell during time $\Delta t$ and the second with one EPSP reaching the cell at time $\Delta_{1}$ (with $\Delta t_{1}+\Delta t_{2}=\Delta t$ ). In the limit of $\Delta t \rightarrow 0$ one can manipulate these expressions to obtain the following differential-difference equation:

$$
\frac{\partial f}{\partial t}=-\frac{\partial}{\partial V}\left(\frac{V}{\tau_{\mathrm{m}}} f\right)+\lambda\left[f\left(V-\Delta V, t \mid V_{0}\right)-f\left(V . t \mid V_{0}\right)\right]
$$

Assuming that the amplitude of a single EPSP is small compared to the threshold $V_{\mathrm{t}}\left(V_{\mathrm{t}} / \Delta V \approx 60\right)$, equation $C .2$ is expanded in a Taylor series. Keeping the first two terms leads to the Fokker-Planck equation:

$$
\frac{\partial f}{\partial t}=-\frac{\partial}{\partial V}\left[A_{1}(V) f\right]+\frac{1}{2} \frac{\partial^{2}}{\partial V^{2}}\left[A_{2}(V) f\right]
$$

where $A_{1}(V)=-V / \tau_{\mathrm{m}}+\lambda \Delta V$ in the first term accounts for the deterministic part (constant mean current and leakage) while $A_{2}(V)=\sigma^{2}=\lambda \Delta V^{2}$ is the variance of the input stream and accounts for the fluctuations. The associated steady-state distribution $W(V)$ is then

$$
w(v)=\frac{1}{\sqrt{\pi \sigma^{2} \tau_{\mathrm{m}}}} e^{-\frac{\left|v-\lambda \Delta V \tau_{\mathrm{m}}\right|^{2}}{\sigma^{2} r_{\mathrm{m}}}}
$$


The mean interspike interval (first-passage-time) is then obtained using Siegert's recursion formula (Ricciardi 1977):

$$
T_{\text {spike }}=\int_{0}^{V_{1}} 2\left[A_{2}(z) W(z)\right]^{-1} d z \int_{0}^{z} W(y) d y
$$

Joint application of equations C.3-C.5 leads to equation 2.4.

\section{Acknowledgments}

We wish to thank Ernst Niebur and William Softky for helpful comments. The research was supported by the Air Force Office of Scientific Research, the Office of Naval Research and the National Institute of Mental Health. Marius Usher was supported by a Myron A. Bantrell Research Fellowship.

\section{References}

Abbott, L. F. 1990. A network of oscillators. J. Phys. A 23(16), 3835-3859.

Abbott, L. F, and van Wresswijk, C. 1993. Asynchronous states in networks of pulse-coupled oscillators. Phys. Rev. E 48(2), 1483-1490.

Abeles, M. 1982. Role of the cortical neuron: Integrator or coincidence detector? Israel J. Med. Sci. 18, 83-92.

Abeles, M. 1991. Corticonics-Neural Circuits of the Cerebral Cortex. Cambridge University Press, Cambridge.

Aertsen, A. M. H. J., Gerstein, G. L., Habib, M. K., and Palm, G. 1989. Dynamics of neuronal firing correlation: Modulation of effective connectivity. J. Neurophysiol. 61(5), 900-917.

Bair, W., Koch, C., Newsome, W., and Britten, K. 1993. Power spectrum analysis of MT neurons in the awake monkey. In Computation and Neural Systems 92, F. Eeckman, ed. Kluwer Academic Publishers, Boston, pp. 495-502.

Bernander, Ö., Douglas, R. J., Martin, K. A. C., and Koch, C. 1991. Synaptic background activity influences spatiotemporal integration in single pyramidal cells. Proc. Natl. Acad. Sci. U.S.A. 88, 11569-11573.

Bernander, Ö., Douglas, R. J., and Koch, C. 1992. A model of cortical pyramidal neurons. CNS memo 16, California Institute of Technology, Pasadena, CA, 91125.

Bush, P. C., and Douglas, R. J. 1991. Synchronization of bursting action potential discharge in a model network of neocortical neurons. Neural Comp. 3, 19-30.

Connors, B. W., and Gutnick, M. J. 1990. Intrinsic firing patterns of diverse neocortical neurons. Trends Neurosci. 13(3), 99-104.

Crick, F., and Koch, C. 1990. Towards a neurobiological theory of consciousness. Sem. Neurosci. 2, 263-275.

Crick, F., and Koch, C. 1992. The problem of consciousness. Sci. Am. 267(3), 152-159. 
Douglas, R. J., Martin, K. A. C., and Whitteridge, D. 1991. An intracellular analysis of the visual responses of neurones in cat visual cortex. J. Phy/siol. 440, 659-696.

Eckhorn, R., Bauer, R., Jordan, W., Brosch, M., Kruse, W., Munk, M., and Reitböck, H. J. 1988. Coherent oscillations: A mechanism of feature linking in the visual cortex? Biol. Cybern. 60, 121-130.

Engel, A. K., König, P., Kreiter, A. K., and Schillen, T. B. 1992. Temporal coding in the visual cortex-new vistas on integration in the nervous system. Thends Neurosci. 15(6), 218-226.

Freeman, W. J. 1975. Mass Action in the Neroous System. Academic Press, New York.

Gray, C. M., and Singer, W. 1989. Stimulus-specific neuronal oscillations in orientation columns of cat visual cortex. Proc. Natl. Acad. Sci. U.S.A. 86, $1698-1702$.

Gray, C. M., König, P., Engel, A. K., and Singer, W. 1989. Oscillatory responses in cat visual cortex exhibit inter-columnar synchronization which reflects global stimulus properties. Nature (London) 338, 334-337.

Hansel, D., and Sompolinsky, H. 1992. Synchronization and computation in a chaotic neural network. Plyys. Rev. Lett. 68(5), 718-721.

Knight, B. 1972. Dynamics of encoding in a population of neurons. J. Gen. Phys. 59, 734-766.

Koch, C., and Schuster, H. 1992. A simple network showing burst synchronization without frequency locking. Neural Comp. 4, 211-223.

Kreiter, A. K., and Singer, W. 1992. Oscillatory neuronal responses in the visual cortex of the awake macaque monkey. Euro. J. Neurosci. 4, 369-375.

Larkman, A. U. 1991. Dendritic morphology of pyramidal neurones of the visual cortex of the rat: I. Branching patterns. J. Comp. Neurol. 306, 307-319.

Llinás, R. 1988. The intrinsic electrophysiological properties of mammalian neurons: Insights into central nervous system function. Science 242, 1654 1664.

Manor, Y., Koch, C., and Segev, I. 1991. Effect of geometrical irregularities on propagation delay in axonal trees. Biophys. J. 60, 1424-1437.

McCormick, D. A., Connors, B. W., Lighthall, J. W., and Prince, D. A. 1985. Comparative electrophysiology of pyramidal and sparsely spiny stellate neurons of the neocortex. J. Neurophysiol. 54(4), 782-806.

Milner, P. M. 1974. A model for visual shape recognition. Psychol. Retr. 81, 521-535.

Mirollo, R. E., and Strogatz, S. H. 1990. Synchronization of pulse-coupled biological oscillators. Siam J. Appl. Math. 50(6), 1645-1662.

Murthy, V. N., and Fetz, E. E. 1993. Effects of input synchrony on the response of a model neuron. In Computation and Neural Systems 92, F. Eeckman, ed. Kluwer Academic Publishers, Boston, pp. 475-479.

Nelson, J. I., Salin, P. A., Munk, M.H.-J., Arzi, M., and Bullier, J. 1992. Spatial and temporal coherence in cortico-cortical connections: A cross-correlation study in areas 17 and 18 in the cat. Vis. Neurosci. 9, 21-37.

Ricciardi, L. M. 1977. Diffusion Processes and Related Topics in Biology. SpringerVerlag, Berlin. 
Softky, W. 1994. Sub-millisecond coincidence detection in active dendritic trees. I. Neurosci. 58(1), 13-41.

Softky, W., and Koch, C. 1993. The highly irregular firing of cortical cells is inconsistent with temporal integration of random EPSP's. J. Neurosci. 13(1), 334-350.

Sompolinsky, H., Golomb, D., and Kleinfeld, D. 1991. Cooperative dynamics in visual processing. Phys. Rev. A 43(12), 6990-7011.

Tononi, G., Sporns, O., and Edelman, G. M. 1992. Reentry and the problem of integrating multiple cortical areas: Simulation of dynamic integration in the visual system. Cerebral Cortex 2, 310-335.

Toyama, K., Kimura, M., and Tanaka, K. 1981a. Cross-correlation analysis of interneuronal connectivity in cat visual cortex. I. Neurophysiol. 46(2), 191201.

Toyama, K., Kimura, M., and Tanaka, K. 1981b. Organization of cat visual cortex as investigated by cross-correlation technique. I. Neurophysiol. 46(2), 202-214.

Ts'o, D. Y., Gilbert, C. D., and Wiesel, T. N. 1986. Relationships between horizontal interactions and functional architecture in cat striate cortex as revealed by cross-correlation analysis. J. Neurosci. 6(4), 1160-1170.

Usher, M., Schuster, J., and Niebur, E. 1993. Dynamics of populations of integrate of fire neurons, partial synchronization and memory. Neural Comp. 5(4), 570-586.

von der Malsburg, C. 1981. The correlation theory of brain function. Internal Report 81(2).

von der Malsburg, C., and Schneider, W. 1986. A neural cocktail-party processor. Biol. Cybern. 54, 29-40.

Received March 4, 1993; accepted July 29, 1993. 